\title{
Cyber-Physical Optimization for Unmanned Aircraft Systems
}

\author{
Justin M. Bradley* and Ella M. Atkins \\ University of Michigan, Ann Arbor, Michigan 48109 \\ DOI: $10.2514 / 1.1010105$
}

\begin{abstract}
Future small unmanned aircraft systems will require careful codesign over both physical and cyber elements to maximize total system efficiency. Mission objectives and success of the system as a whole are becoming increasingly dependent on appropriate allocation of computational resources balanced against demands of the physical actuation systems. In this paper, a cooptimization scheme is described that considers tradeoffs between costs associated with the physical actuation effort required for control and the computational effort required to acquire and process incoming information. A small unmanned aircraft system surveillance mission, the visual inspection of a pipeline, is proposed to investigate specifics of cyber-physical cost terms and their tradeoffs. A multidisciplinary cost function minimizes energy and maximizes mission efficiency and effectiveness. Pareto fronts are examined over combinations of competing cyber and physical objectives, and they demonstrate that excluding either cyber or physical cost terms results in reduced performance for the holistic system over the course of the mission.
\end{abstract}

\section{Nomenclature}

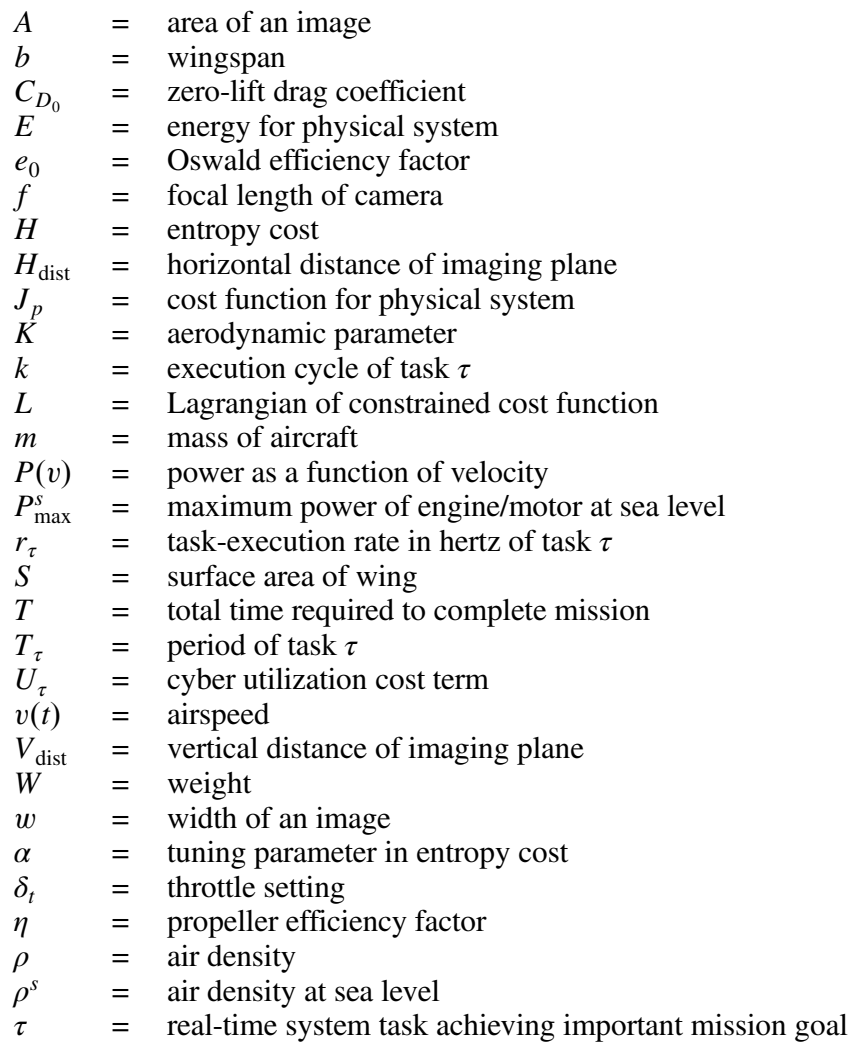

\section{Introduction}

$\mathbf{T}$ HE primary goal of air vehicle design and operation has historically been to achieve appropriate maneuverability and to overcome aerodynamic drag and the influence of gravity in a manner that maximizes range and/or endurance. To date, the energy a powered aircraft requires to apply the necessary propulsive and control actuation forces over a flight has dominated the total energy consumed across all other vehicle subsystems. Surveillance unmanned aircraft systems (UASs) [1] are becoming smaller and are constructed with composite materials that minimize weight. They are also being equipped with increasingly sophisticated avionics and payloads. Powered glider designs in particular exhibit low drag and weight, resulting in a significantly reduced thrust requirement. For the first time, the power required by avionics and payload systems for a flight vehicle is comparable to propulsive plus control actuation requirements. We will likely see a future where avionics and payload

Presented as Paper 2012-2447 at the Infotech@Aerospace, Garden Grove, CA, 19-21 June 2012; received 20 December 2012; revision received 2 May 2013; accepted for publication 4 May 2013; published online 31 January 2014. Copyright $\odot 2013$ by Justin Bradley. Published by the American Institute of Aeronautics and Astronautics, Inc., with permission. Copies of this paper may be made for personal or internal use, on condition that the copier pay the $\$ 10.00$ per-copy fee to the Copyright Clearance Center, Inc., 222 Rosewood Drive, Danvers, MA 01923; include the code 2327-3097/14 and \$10.00 in correspondence with the CCC.

*Ph.D. Student, Aerospace Engineering Department. Student Member AIAA.

${ }^{\dagger}$ Associate Professor, Aerospace Engineering Department. Associate Fellow AIAA. 
power can even exceed power required for force application, particularly during periods of demanding onboard processing and communication activity.

Control systems engineers typically optimize vehicle trajectories, and thereby force application time histories over physics-based models of vehicle dynamics, including flight envelope and actuator saturation constraints. Conversely, software engineers optimize processor and communication resource use over the suite of computational and information-sharing tasks, regulating energy use through the real-time regulation of variable-speed processors, activation/shutdown of cores in a multiprocessor system, and regulation of communication links. While real-time task-execution models are typically discrete rather than continuous time, the methods used to optimally control physical and real-time computing systems are fundamentally the same: gradient- or search-based algorithms are used to identify minimum-cost solutions given constraints.

For emerging UASs that consume comparable power for avionics versus force application, or for which the mission effectiveness is dependent on both cyber and physical resources, neither physical- nor cyber-system optimization is dominant. Therefore, globally optimal (minimum energy, minimum time, maximum information) performance can only be achieved if cyber and physical models can be shown independent of each other, or if necessary cyber and physical couplings are identified and simultaneously considered during optimization. Computational resources must be used as a minimum to guide, navigate, and control the UASs as well as to compute or update future spatiotemporal (four-dimensional) trajectories. Physical trajectories in turn enable the cyber system to maximize its ability to acquire information (e.g., from sensors) and to communicate (e.g., with ground operators). Cyber and physical resources are therefore necessarily coupled.

This paper presents a new multidisciplinary optimization [2] direction for which the models being integrated optimize energy consumption over both physical effectors and computational (cyber) resources. The cost function to be optimized includes weighted terms representing energy used by physical actuators and energy used by a multicore or variable-speed processing architecture. This paper presents a case study of a UAS surveillance mission aimed at assessing the potential performance improvements possible with cooptimization of cyber and physical resources over energy use, time, and mission accomplishment. A mission-appropriate analytical cost function is developed to provide a minimal-cost trajectory over the mission. We simplify the cost function by allowing design variables to remain static throughout the mission, consistent with a steady flight scenario, thereby reducing the complexity of the cost function and optimization process. We then examine Pareto fronts for combinations of cost function objectives to demonstrate the important tradeoffs between physical and cyber resources and to give insight into the interdependence between them. We use a numerical solver to find physical-subsystem optimal, cyber-subsystem optimal, and holistic-system optimal solutions, and we compare them with solutions selected from Pareto front analysis. We demonstrate that only via a total cyber-physical system (CPS) optimization can one achieve efficiency throughout the total system.

For our case study, we adopt a solar-supplemented powered-glider small UAS currently flown by a University of Michigan student team (SolarDrones $\$$ ) for which steady flight performance parameters are available. The small UAS payload is a downward-facing video camera that can provide frames at a variable rate. The simulated avionics allow direct regulation of computational power requirements in a manner that trades energy use with camera data-acquisition bandwidth. A one-dimensional pipeline inspection case study is investigated, focusing attention on physical and computational energy use tradeoffs without complexity in the actual path through three-dimensional space.

\section{Related Work}

The design of control systems under the constraints of real-time computational (cyber) resources has been extensively studied. Anytime control [3-5] tries to improve controller accuracy as a function of available cyber resources. In feedback scheduling [6-8], cyber-resource allocation is modified in real time according to the evolving needs of the tasks requiring these resources. Despite these advances, most deployed aerospace systems still execute static schedules for cyber-resource use. In safety-critical commercial systems, these schedules execute on real-time operating systems and have been analyzed offline to show hard task deadlines are met. In lower-cost systems (e.g., small UAS), code execution rates may be ad hoc, with code running "as quickly as possible" on a non-real-time operating system such as an embedded Linux distribution. Such a simple execution strategy can be successful so long as tasks sufficiently underutilize available cyber resources in the worst case, as is typically the case when "fly-to-waypoint" guidance, navigation, and control system logic and low-bandwidth ground station communication are all that executes onboard. As more sophisticated logic such as image-processing and flight-plan optimization algorithms migrate onboard to enable robustness to scenarios such as lost link and/or loss of GPS, resources will no longer be underutilized, and thus must be carefully managed in real time.

For satellite systems, target information gathering by imaging systems generally must occur within in a relatively short time window [approximately $3 \mathrm{~min}$ for low Earth orbit (LEO)], during which the system must maximize its efforts to collect the data. There is generally a 10 to 15 min window during which the system can prepare resources for intense data-collection periods. Traditionally, such task-scheduling problems have been addressed by ground operators manually constructing plans with write and check procedures [9]. However, automated methods have been proposed and used with success. Bataille et al. examined and designed for physical constraints, fairness, and efficiency for different agents using a shared resource (an Earth-observing satellite) [10]. In work by Bresina et al. two techniques, GenH, which generates a specialized search heuristic, and heuristic-biased stochastic sampling, which employs the heuristic within a stochastic sampling method, are combined together to automatically generate high-quality schedules with respect to an objective function [11]. EO-1 is the first of a series of NASA missions entitled "Earth Observer (EO)" targeting both science and technology demonstration goals. The continuous activity scheduling, planning, execution, and replanning (CASPER) planner was used onboard EO-1 to optimize science activities based on incoming data. An iterative repair algorithm was used to improve the task-execution schedule. This science planner was highly successful, and it has continued to evolve for infusion into additional missions. Despite this success, mission directors have not relied on CASPER for scheduling safety-critical guidance, navigation, and control (GNC) tasks because of their lack of trust in automation that is difficult to fully validate and verify due to software and abstract model complexity issues. Such trust will be established as dynamic planning/scheduling systems such as CASPER continue to operate reliably and continue to provide more and better data than would be possible without such automation. Because architectures such as CASPER have not had the opportunity to schedule GNC along with science tasks, the CPS optimization proposed in this paper has not been studied by the space science community [12].

Agrawal et al. explored some of the reasons why more advanced control algorithms are not used in modern aircraft and spacecraft avionics system [13]. They concluded that a quality of service (QOS) approach [14] is needed to address the problem, and they proposed an adaptive resource-management scheme for a real-time avionics system using anytime control and accompanying nontraditional task scheduling. Abdelzaher et al. [14] and Atkins and Sanner [15] previously investigated the use of QOS negotiation for guidance, navigation, and control. Russ and Stütz recently proposed a higher-level style of resource management that includes task-based guidance, navigation, and perception plans [16]. Their method focuses on finding algorithmic solutions adapting to perceptual demands that vary during flight as well as balancing those demands

${ }^{\ddagger}$ Data available online at http://solarbubbles.engin.umich.edu/ solarbubbles/ [retrieved 2013]. 
with sensory and computational resources [16]. Narayan et al. presented a novel, computationally adaptive, trajectory decision optimization system that can dynamically manage, calculate, and schedule task-execution parameters [17]. Offline and online components work together to increase overall mission efficiency.

Our work complements existing research by providing a mechanism to optimize over cyber and physical resources while leveraging optimization theory and, more particularly, optimal control [18]. Existing solutions to dynamically adjust parameters can provide the tools by which a system could use our methodology to produce more efficient missions according to the individual metrics chosen in the cost function. We also hope to combine the work presented in this paper with our previous work in [19], in which we balance mission-critical cyber tasks with physical system performance, leading to further progress toward a well-rounded codesign process for CPS.

\section{Cost Functions}

We seek optimization over both physical and cyber characteristics of the UAS and its mission in order to more holistically optimize system performance. This means developing cost terms for task performance and energy required for cyber activities as well as for control actuation effort and propulsion. Moreover, we naturally want to maximize efficiency of our designated mission, which will include goals for both the physical-and cyber components of the UAS. These mission-dependent goals may include maximizing coverage area or amount of information acquired for a given area, along with collection, processing, and transmission of data.

In this work, we develop cost terms representing both mission goals and efficiency for a UAS for which the mission is surveillance of a straight section of pipeline with a small, lightweight downward-facing gimballed camera. We focus on movement in one dimension only and assume flight dynamics are governed by steady-flight assumptions.

Our objective for the physical system will be to determine the optimal velocity (airspeed in one dimension) of the UAS for the mission. Owing to the assumptions of the mission and steady flight, we rely on a gimbal to consistently adjust the camera to point directly toward the ground (optical axis perpendicular to ground plane), which compensates for changes in pitch of the aircraft needed to accommodate various velocities of flight.

We model a single real-time task to accomplish the primary goals of the mission related to pipeline surveillance. This task performs image acquisition, processing, and communication/storage of image. Our design objective for the cyber system will be to determine the optimal execution rate of this task. While there are other system-critical tasks on the cyber system, including the control task, we assume these require a fixed amount of resources. We instead focus on optimizing over the remaining noncritical bandwidth available in the cyber system.

We divide the cost terms into physical and cyber goals for clarity, and we emphasize the assimilation of each into a systemwide cost function. "Physical" in the context of a UAS includes items related to flight: for example, the airframe, propulsion system, and control surfaces. "Cyber" relates to items required for data processing, communication, image collection, computation of control inputs, etc. In this work, we endeavor to focus clearly on the idea of combining physical and cyber cost terms into a holistic cyber-physical system cost function.

\section{A. Physical System Terms}

Small UASs often have very modest energy reserves, most often consisting of small battery packs or a small fuel tank. In non-energy-harvesting applications under normal conditions, such energy supplies can provide between 30 min to multiple hours of flight time. These flight times can be reduced when cyber-intensive activities such as image processing and communication are involved. Minimizing energy consumption during steady flight is an important consideration in the design and control of the UAS.

\section{Physical System Energy}

In most aircraft applications, propulsion will consume the majority of the energy required for flight, surpassing actuation effort required by control surfaces. For simplicity, in this work, we assume propulsion is the only drain on energy supplies by the physical system and we model steady flight in which power used by control surface servos would be constant or near constant. We therefore seek to minimize energy of the physical system over the entire mission:

$$
E=\int P(v(t)) \mathrm{d} t
$$

where $P(v(t))$ is a traditional model for power as a function of velocity [20]

$$
P(v(t))=\frac{1}{2} S C_{D_{0}} \rho v(t)^{3}+\frac{2 K W^{2}}{\rho v(t) S}
$$

In steady level flight, the power of the aircraft, and therefore velocity, is manipulated by a throttle setting that maps nonlinearly to power as

$$
P=\eta \delta_{t}\left(\frac{\rho}{\rho^{s}}\right)^{m} P_{\max }^{s}
$$

where $P_{\max }^{s}$ is the maximum power of the engine/motor at sea level, $m>0$ is a characteristic of the engine/motor, $0 \leq \eta \leq 1$ is a propeller efficiency factor, $\rho^{s}$ is the air density at sea level, and $0 \leq \delta_{t} \leq 1$ is the throttle setting [20]. The power curve for our UAS (described in Sec. IV.D.1) can be seen in Fig. 1 .

2. Time

In addition to minimizing energy, ideally, we would like to efficiently accomplish our mission by minimizing the time required to complete it. Such time-minimal optimization cost terms appear frequently and are given by

$$
T=\int \mathrm{d} t
$$




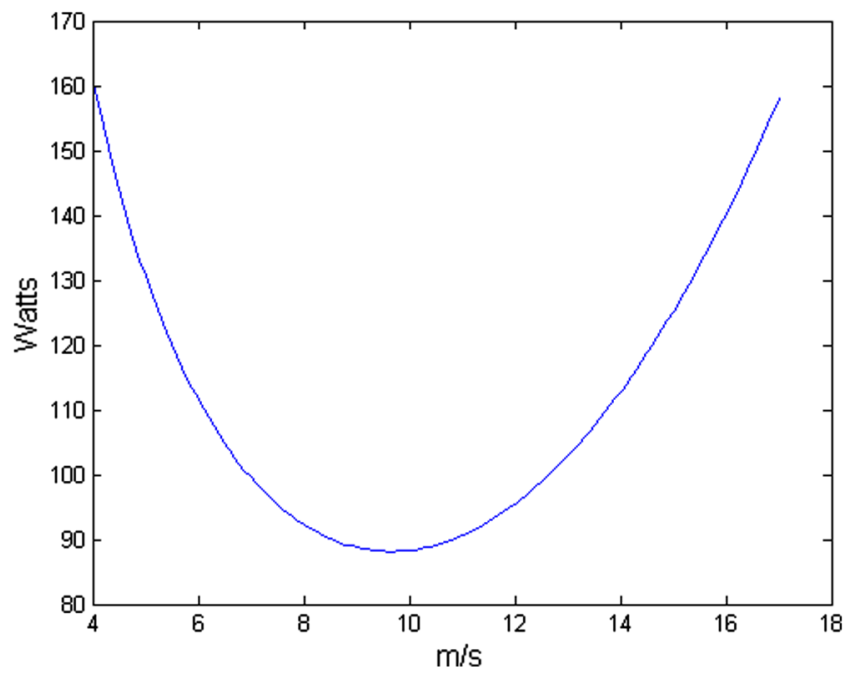

Fig. 1 Power curve for SolarDrones UAS.

3. Cost Function for Physical System

These two competing objectives, $E_{p}$ and $T$, comprise the cost function for the overall physical system:

$$
J_{p}(v(t))=\beta_{p 1} \int P(v(t)) \mathrm{d} t+\beta_{p 2} \int \mathrm{d} t
$$

where $\beta_{p 1}$ and $\beta_{p 2}$ are weighting terms. Optimizing $J_{p}$ alone is what a traditional trajectory or path planner might do if no costs are attributed to the cyber system. While some UAS researchers have added tracking information, target acquisition, and other mission objectives to their control and optimization algorithms [21,22], to our knowledge this has historically been done from the physical perspective without attempting to optimize over cyber-system performance and requirements.

\section{B. Cyber-System Terms}

In a modern, fully autonomous UAS, the cyber system becomes the gateway for virtually all aspects of the system. Control actuation inputs, data collection, communication, throttle setting, and path and mission planning are potentially all being done simultaneously onboard. While realtime system researchers have advanced scheduling techniques for prioritizing each of these critical tasks, the correlation between physical performance, mission objectives, and computational efficiency has remained largely unexplored [23].

In many cyber-physical systems, task-execution rates are selected a priori based on requirements of the system. For example, the sampling rate of the control task may be selected based on digital control analysis, thereby ensuring robustness and stability margins. While it is unreasonable to interfere with such high-priority tasks, lower-priority tasks may still have some flexibility in task-execution rate, allowing us to optimize over mission and cyber parameters without interfering with mission-critical tasks.

In our previous work, we explored the tradeoff of mission critical task-execution rates and physical performance [19,24]. For this work, we assume that hard real-time feedback control tasks are appropriately scheduled and executed while we focus on the rest of the available cyber resources for soft real-time tasks. More specifically, we assume that we cannot only conserve energy by optimally selecting execution rates of lower-priority tasks, but we can also increase mission effectiveness by developing costs that relate task-execution rates to mission efficiency.

\section{Cyber Utilization}

In real-time system scheduling theory, online schedules can be created by examining relative deadlines of independent periodic tasks as in the earliest deadline first scheduling algorithm. Such optimal scheduling algorithms are dynamic in that they can assign task priority as jobs are released to the operating system for scheduling [25]. They therefore have the ability to respond to changing deadlines and periodic rates.

In this work, we assume that at least part of the cyber utilization is fixed based on selected and scheduled periodicity of mission-critical tasks, consistent with current practices in the aerospace community. We then focus on maximizing use of the remaining resources. The innovation of this work relative to applications studied by others (e.g., CASPER for EO-1) is that we purposely cooptimize the speed of the aircraft and the speed of the payload (image) data acquisition/processing. Because aircraft motion changes what is observed in the acquired image sequence, and because energy is consumed by flight control and payload systems, this cooptimization is essential for a more "globally optimal" solution than is possible when separately optimizing cyber- and physical-resource usage.

In this initial cooptimization work, we assume a single task $\tau$ is repeatedly executed to achieve the mission goal of capturing and processing images of a pipeline to be inspected. The task runs at execution rate $r_{\tau}(k) \mathrm{Hz}$ and has a maximum execution rate of $r_{\tau, \text { max }} \mathrm{Hz}$ stemming from restrictions based on available cyber resources and the worst-case execution time (WCET) of task $\tau$. That is, we ensure schedulability of the task based on $r_{\tau \text {, max }}$ but allow that rate to slow down, resulting in freed cyber resources to be devoted to increased service of other processing tasks or to conserving energy through fewer memory cycles, reduced processor clock rate when possible, and/or shutdown of some system cores when possible. Let $k$ represent the execution cycle of $\tau$, incrementing each time task $\tau$ is run. The execution rate of $\tau$ at cycle $k$ is then $r_{\tau}(k)$. Figure 2 demonstrates an example processor utilization timeline depicting $r_{\tau}(k)$. We then introduce the cyber utilization term

$$
U_{\tau}=\sum_{k} \frac{r_{\tau}(k)}{r_{\tau, \max }}
$$

Note that the rate of execution cannot change during a particular execution cycle of that task. We assume that cyber utilization is proportional to energy consumed by the cyber system and, as a result, minimizing it is the cyber equivalent to the energy minimization term of the physical system in Eq. (1). 


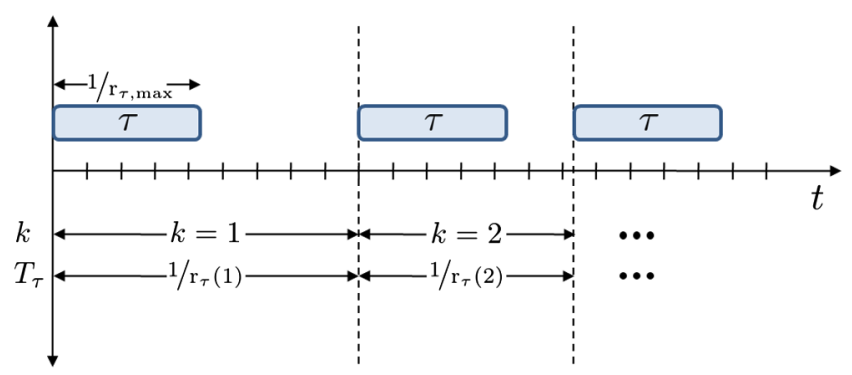

Fig. 2 Processor utilization timeline for task $\tau$.

\section{Mission Information}

We seek to relate mission efficiency to cyber and physical parameters. For our specified mission, we assume that detailed imagery of the pipeline is critical for detecting aberrations and problems. Collecting an appropriate amount of imagery and, more specifically, appropriate imagery of any pipeline cracks, is critical for detection of problems. We approach this goal from an information theory viewpoint and desire to create a cost term in that context.

Information theory was originally designed to be applied to source coding, where the limits of data compression are lower bounded by the entropy of the source code $[26,27]$. However, it has since been applied to numerous fields and in numerous creative ways, including image processing, object detection, and surveillance. In image processing and computer vision, mutual information can be used to provide image registration in medical imaging [28], while maximum entropy is regularly used in image reconstruction, particularly in astronomy [29]. In the context of UAS surveillance systems, information theory has been used for dim target detection in sense-and-avoid applications [30].

The most common quantity of information is entropy of a random variable. Let $X$ be an ensemble $\left\{x, \mathcal{A}_{x}, P_{x}\right\}$, where $x$ are the outcomes, $\mathcal{A}_{x}$ is the domain or sample space, and $P_{x}$ the set of probabilities of outcomes. Then, the entropy for a discrete ensemble is defined as

$$
H(X)=-\sum_{x \in \mathcal{A}_{x}} p(x) \log _{2} p(x)
$$

Intuitively, entropy measures the average unpredictability of a random variable.

Information theory is used in this work to develop an appropriate mission information cost term. To ensure a well-behaved total system cost function, each individual cost function must be continuous, convex, and nonnegative such that, when minimized, greater benefits are realized. This means we need a metric that, when minimized, produces increased information about the pipeline being imaged.

For surveillance missions in which detection of aberrations or events are important, multiple observations of any single point in the area in question are valuable. Acquiring multiple images of the same ground points has the advantage of providing additional viewpoints and redundant data, and it may allow for super-resolved imagery, thereby increasing our ability to detect pipeline anomalies [31]. Keeping this in mind, we propose a cost term based on overlap between successive images where increasing overlap is rewarded. Increased overlap between images is equivalent to lower entropy as the scene in each successive image changes little. Additionally, if there is underlap, each successive image is completely new information about the pipeline, and therefore provides the maximum entropy. From this perspective, contrary to traditional applications of information theory, we seek to minimize entropy, as this strategy provides the most redundant information.

From an information theory perspective, we view information cost as an exponential distribution of redundancy of acquired information of the scene or overflown region (pipeline). In this sense, minimizing the entropy has the effect of maximizing the total information acquired. This term has the effect of requiring a combination of slow aircraft speed and/or increased task frequency, and it depends on both velocity of the aircraft $v(t)$ and the rate of image acquisition and processing $r_{\tau}(k)$ :

$$
H=\int e^{-\alpha \Omega\left(v(t), r_{\tau}(k)\right)} \mathrm{d} t
$$

We let $\alpha$ be a tuning parameter. The overlap between successive image footprints is then

$$
\Omega\left(v(t), r_{\tau}(k)\right)=\frac{1}{A}\left(A-w \int_{t-T_{\tau}(k)}^{t} v(\gamma) \mathrm{d} \gamma\right)
$$

where $A$ is the total area of an image, $w$ is the width of an image, and $T_{\tau}(k)=1 r_{\tau}(k)$ is the period of task $\tau$. An exponential distribution was used, as it provides a rapidly increasing penalty for flying too fast and appropriate diminishing returns for flying slowly. For simplicity, we assume that the aircraft flies at approximately the same height above ground for the duration of the mission; therefore, $A$ and $w$ remain constant. In Fig. 3 , we show a plot of $H$ to demonstrate how the entropy changes with both cyber rate $r_{\tau}$ and velocity $v$. The dependence of entropy on cyber rate falls off as a steep exponential, and it falls off more gradually with aircraft velocity. This means we expect our Pareto front analysis in Sec. V.A to indicate that lower entropy will be achieved primarily by increasing cyber rate rather than flying at a slower velocity.

3. Cost Function for Cyber System

The expressions in Eqs. $(\underline{6}, \underline{8})$ comprise the cost function for the cyber system:

$$
J_{c}\left(v(t), r_{\tau}(k)\right)=\beta_{c 1} \sum_{k} \frac{r_{\tau}(k)}{r_{\tau, \max }}+\beta_{c 2} \int e^{-\alpha \Omega\left(v(t), r_{\tau}(k)\right)} \mathrm{d} t s
$$

where we have weighting terms $\beta_{c 1}$ and $\beta_{c 2}$. Such a cost function might be used if we were only interested in trading cyber-resource utilization cost against reward for accomplishing mission objectives, which in the pipeline inspection case study maps to minimizing entropy (increasing information redundancy) that could be obtained through overlapping image data acquisition and processing. 


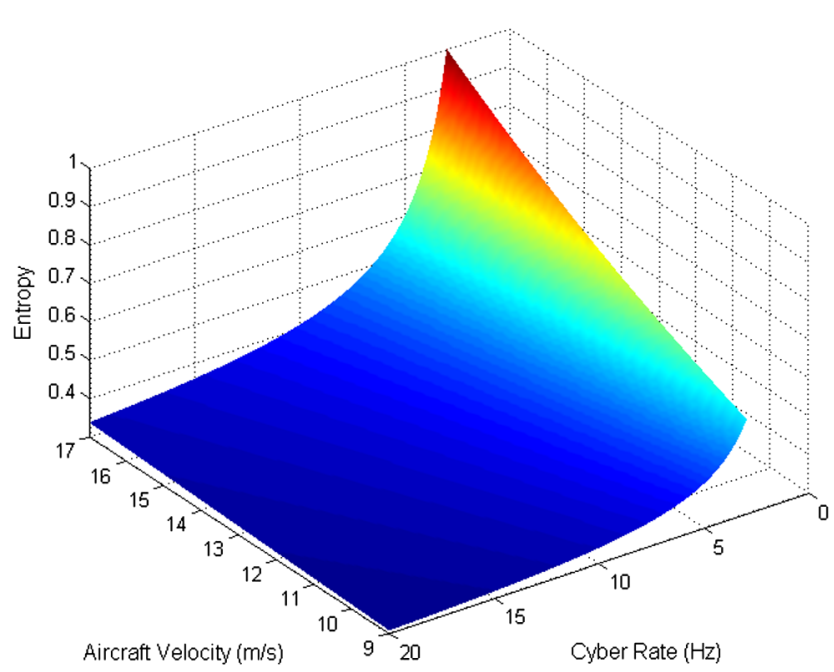

Fig. 3 Entropy cost $H$.

\section{CPS Cost Function}

We combine $J_{p}$ and $J_{c}$ to obtain a holistic CPS cost function:

$$
J\left(v(t), r_{\tau}(k)\right)=\beta_{p 1} \int P(v(t)) \mathrm{d} t+\beta_{p 2} \int \mathrm{d} t+\beta_{c 1} \sum_{k} \frac{r_{\tau}(k)}{r_{\tau, \max }}+\beta_{c 2} \int e^{-\alpha \Omega\left(v(t), r_{\tau}(k)\right)} \mathrm{d} t
$$

In Sec. V, we will use appropriate weighting to compare physical-only optimization, cyber-only optimization, and total system optimization to demonstrate how increased efficiency and conservation of energy can be achieved by including both physical and cyber objectives.

\section{Setup and Solution}

Our mission objective is to survey a straight segment of pipeline by flying a small high-aspect-ratio UAS with a downward-facing gimballed camera directly overhead. We have created a simulation in MATLAB to compare various solutions to the optimization problem posed. We first list the assumptions we have made to simplify the problem and demonstrate why an analytical solution is not possible. We then describe the numerical methods chosen to solve our optimization problem, and we discuss the models we have adopted.

\section{A. Assumptions}

Equation (11) is nontrivial to solve, in part due to the need to find the time-varying solution $v(t)$ and $r_{\tau}(k)$. It is further complicated by the discrete nature of the cyber-system design variable $r_{\tau}(k)$, making this a mixed discrete-continuous equation. We make the following assumptions in order to simplify the problem: Assumption 1: The segment of pipeline is straight.Assumption 2: We assume aircraft performance is consistent with the principles of steady level flight.Assumption 3: The mission takes place close to sea level, with a relatively low altitude allowing use of standard sea level air density.Assumption 4: Altitude remains approximately constant through the mission.Assumption 5: Due to assumptions 1 and 2, the onboard gimballed camera always points straight down toward the ground. Specifically, this implies the optical axis of the camera is always perpendicular to the ground plane.Assumption 6: The scene, the ground, and the accompanying pipeline are approximately flat compared with the camera's height.Assumption 7: We restrict our problem to finding the optimal static $v$ and $r_{\tau}$ that minimize the cost of the mission assuming $v(t)$ and $r_{\tau}(k)$ remain constant throughout.

An interesting addition to this work to be made in the future will be to model certain places on the pipeline as "high interest," either a priori or through real-time image processing, and therefore solve for the optimal trajectory with dynamically changing velocity and task-execution rate.

\section{B. Simplified Cost Function}

Let $r_{\tau \text { max }}$ be the WCET of task $\tau$ and $D$ be the (constant) total straight-line distance of the entire mission. Based on the assumptions made, and knowing the total distance $D$ of the mission, we can rewrite the overlap term $\Omega\left(v(t), r_{\tau}(k)\right)$ as

$$
\Omega\left(v, r_{\tau}\right)=1-\frac{w v}{r_{\tau} A}
$$

where $w$ and $A$ denote the constant width and area of an image footprint, respectively. Because we limit our problem to finding the optimal static $v$ and $r_{\tau}$, and owing to the fixed distance of the mission, we can replace the integrals in Eq. (11) with the total corresponding quantities as a function of $v$ and $r_{\tau}$. This yields

$$
J\left(v, r_{\tau}\right)=\beta_{p 1} \frac{D P(v)}{v}+\beta_{p 2} \frac{D}{v}+\beta_{c 1} \frac{r_{\tau}}{r_{\tau, \max }}+\beta_{c 2} D e^{-\alpha \Omega\left(v, r_{\tau}\right)}
$$

In Fig. $\underline{4}$ is a plot of $J\left(v, r_{\tau}\right)$ in Eq. (12). We note the convex shape and unconstrained nature of the minimum, implying we should obtain a robust solution with an appropriate numerical optimization method.

A difficulty in any optimization scheme is the correct selection of weights for each cost metric. To equalize the contribution from each term, we normalize each, thereby giving us more intuition later on as we choose our weighting terms $\beta_{p 1}, \beta_{p 2}, \beta_{c 1}$, and $\beta_{c 2}$. Because we have constrained our problem to realistic parameters for velocity $v$ and mission task rate $r_{\tau}$, we can compute $\max \{E\}$ and $\max \{T\}$, occurring at the slowest velocity 


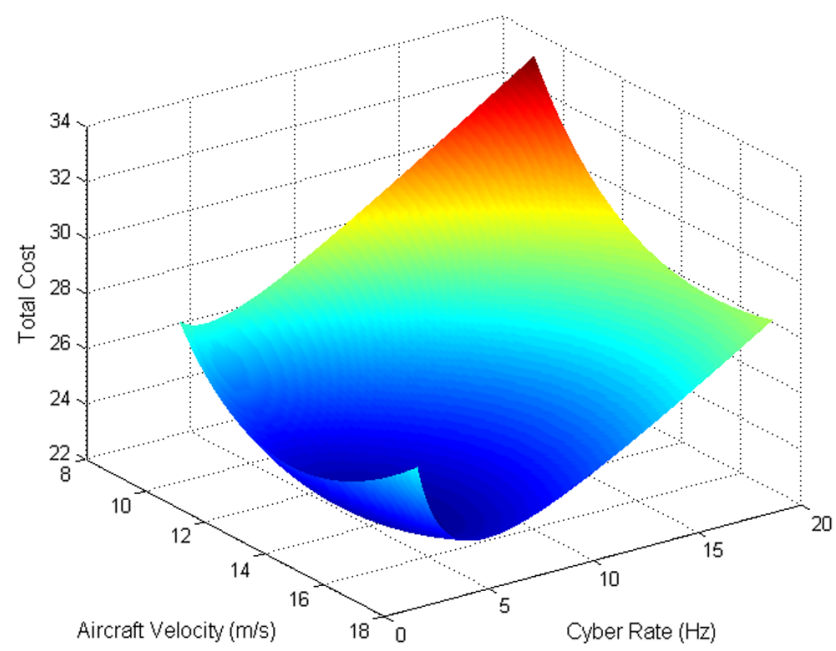

Fig. $4 J\left(v, r_{\tau}\right)$.

$\left(v_{\min }\right)$, and $\max \{H\}$ occurring under conditions giving rise to lowest amount of overlap between images $\left(v_{\max }, r_{\tau, \min }\right)$. Combining these, we obtain a new normalized cost function:

$$
J\left(v, r_{\tau}\right)=\beta_{p 1} \frac{D P(v)}{v \max \{E\}}+\beta_{p 2} \frac{D}{v \max \{T\}}+\beta_{c 1} \frac{r_{\tau}}{r_{\tau, \max }}+\beta_{c 2} \frac{D e^{-\alpha \Omega\left(v, r_{\tau}\right)}}{\max \{H\}}
$$

After substitutions and some algebra, we can rewrite Eq. (13) as

$$
J\left(v, r_{\tau}\right)=\beta_{p 1} \gamma_{1} v^{2}+\frac{\beta_{p 1} \gamma_{2}}{v^{2}}+\frac{\beta_{p 2} v_{\min }}{v}+\frac{\beta_{c 1} r_{\tau}}{r_{\tau, \max }}+\beta_{c 2} \gamma_{3} e^{w v / r_{\tau} A}
$$

where

$$
\begin{gathered}
\gamma_{1}=\frac{v_{\min } S C_{D_{0}} \rho}{2 P\left(v_{\min }\right)} \\
\gamma_{2}=\frac{2 v_{\min } K W^{2}}{\rho S P\left(v_{\min }\right)} \\
\gamma_{3}=\frac{e^{-\alpha}}{e^{-\alpha \Omega\left(v_{\max }, r_{t, \min }\right)}}
\end{gathered}
$$

\section{Analytical Solution and Feasibility}

We investigated the possibility of identifying a minimum for Eq. (14) through analytical computation. Due to the constraints of the flight envelope, and of the real-time computing system, let domain $\mathcal{D} \subset \mathbb{R}^{2} \overline{\text { be }}$

$$
\mathcal{D}=\left\{\left[\begin{array}{c}
v \\
r_{\tau}
\end{array}\right] \in \mathbb{R}^{2} \mid\left[\begin{array}{c}
v_{\text {min }} \\
r_{\tau, \min }
\end{array}\right] \leq\left[\begin{array}{c}
v \\
r_{\tau}
\end{array}\right] \leq\left[\begin{array}{c}
v_{\text {max }} \\
r_{\tau, \max }
\end{array}\right]\right\}
$$

which is a compact set. From the Weierstrass theorem, $J\left(v, r_{\tau}\right)$ in Eq. (14) does have a global minimize..** In attempting to find an analytical solution, we formulate the constrained optimization problem

$$
\begin{gathered}
\text { Minimize } J\left(v, r_{\tau}\right) \\
\text { subject to } v \leq v_{\max } \\
v \geq v_{\min } \\
r_{\tau} \leq r_{\tau, \max } \\
r_{\tau} \geq r_{\tau, \min }
\end{gathered}
$$

\footnotetext{
**Bernstein, D. and Tsiotras, P., "A Course in Classical Optimal Control," Unpublished Notes, Version: 15 June 2009, 2009.
} 


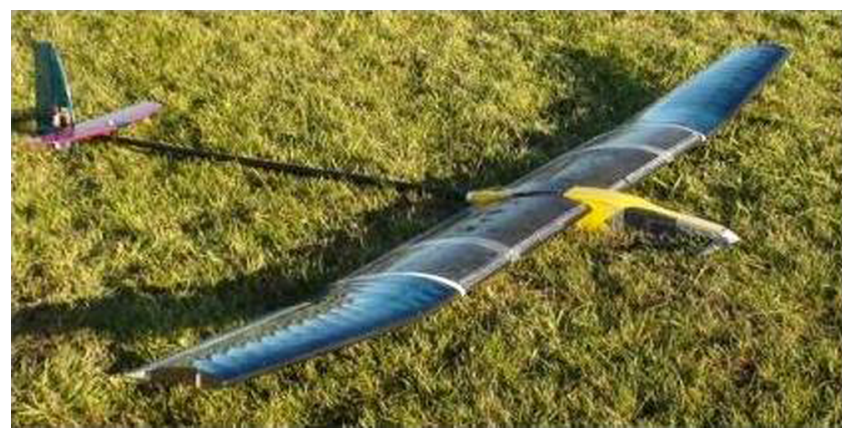

Fig. 5 SolarSight solar-powered UAS.

To solve the constrained optimization problem, we form the Lagrangian

$$
L\left(v, r_{\tau}, \lambda_{1 \ldots 4}\right)=J\left(v, r_{\tau}\right)+\lambda_{1}\left(v-v_{\max }\right)+\lambda_{2}\left(-v+v_{\min }\right)+\lambda_{3}\left(r-r_{\tau, \max }\right)+\lambda_{4}\left(-r+r_{\tau, \min }\right)
$$

where $J\left(v, r_{\tau}\right)$ is from Eq. (14), and $\lambda_{1 \ldots 4}$ are Lagrange multipliers. However, in applying the Karush-Kuhn-Tucker necessary conditions to the Lagrangian, we encounter a transcendental function as part of a set of equations with no analytical solution:

$$
\begin{gathered}
\nabla_{v} L=2 \beta_{p 1} \gamma_{1} v-\frac{2 \beta_{p 1} \gamma_{2}}{v^{3}}-\frac{\beta_{2} v_{\min }}{v^{2}}+\frac{w \beta_{c 2} \gamma_{3}}{r_{\tau} A} e^{w v / r_{\tau} A}+\lambda_{1}-\lambda_{2} \\
\nabla_{r_{\tau}} L=\frac{\beta_{c 1}}{r_{\tau, \max }}-\frac{w v \beta_{c 2} \gamma_{3}}{A r_{\tau}^{2}} e^{w v / r_{\tau} A}+\lambda_{3}-\lambda_{4}
\end{gathered}
$$

Even in the case where weights are chosen to eliminate any active constraints, the set of equations in Eq. (18) has no analytical solution. This requires us to resort to numerical solutions.

\section{Experimental Models and Setup}

\section{Aircraft}

The SolarDrones student team in the Aerospace Engineering Department at the University of Michigan has designed, built, and tested a solarsupplemented powered-glider UAS. Their latest platform, SolarSight (seen in Fig. 5), has been modeled as part of the design process. In our simulation, we presume the aerodynamic and power model parameters given in Table 1 . This model leverages the well-known power/velocity relationship of a single-engine propeller-driven aircraft, as was described in Eq. (2) [20]. From these model parameters, we compute the remaining necessary parameters for the power equation, which are shown in Table $\underline{2}$.

\section{Camera}

For the camera, we use a standard pinhole model consistent with specifications of the Panasonic GP-CX161/45P/E. $\frac{\S}{\mathrm{s}} \mathrm{The}$ key parameters for this camera are shown in Table 3. Given the pinhole assumption for simplicity, we do not model lens distortions and other effects. We also assume that the ground and pipeline are approximately flat compared to the much larger camera height above the ground. Because we know the camera height above the ground at all times, presumed constant in steady level flight conditions, we can directly calculate the image footprint on the ground as a function of height above the ground. This is done by projecting the four corners of the image plane onto the (presumed) flat ground forming the image footprint $[\underline{32}, \underline{33}]$. We can then easily compute overlapping area between acquired images.

\section{Experimental Setup}

For the SolarSight aircraft, the stall speed (around $9 \mathrm{~m} / \mathrm{s}$ ) and maximum power output of the engine/motor determine the bounds of $v$. For the cyber rate $r_{\tau}$, the lower bound was chosen based primarily on tuning the information cost in Eq. (8). That is, at rates lower than $3 \mathrm{~Hz}$, there was no overlap between images resulting in coverage gaps; thus, maximum entropy $(H=1)$ is presumed for the entire mission. The maximum cyber rate was chosen based on diminishing returns from cyber rates higher than $20 \mathrm{~Hz}$. Bounds are therefore

$$
9 \mathrm{~m} / \mathrm{s} \leq v \leq 17 \mathrm{~m} / \mathrm{s}
$$

$$
3 \mathrm{~Hz} \leq r_{\tau} \leq 20 \mathrm{~Hz}
$$

Additionally, via tuning, we chose the parameter $\alpha=4$ in Eq. (13), and we chose the height above the ground (from which we are able to derive $A_{i}$ and $w_{i}$ ) to be $30 \mathrm{~m}$.

In our simulation, we used MATLAB's fmincon function to solve the optimization problem. There are a variety of available algorithms, and we obtain equally good results with fmincon's implementation of the active-set and sequential quadratic programming algorithms. $\mathbb{1}$

${ }^{\S}$ Data available online at http://www.hicam.com/pana/Pana.pdf [retrieved 2013].

"Data available online at http://www.mathworks.com/help/toolbox/optim/ug/fmincon.html [retrieved June 2012]. 
Table 1 UAS model parameters

\begin{tabular}{ccc}
\hline \hline Parameter & Description & Value \\
\hline$e_{0}$ & Oswald factor & 0.95 \\
$b$ & Wingspan & $3.3 \mathrm{~m}$ \\
$S$ & Surface area & $1.0 \mathrm{~m}^{2}$ \\
$m$ & Mass & $11.5 \mathrm{~kg}$ \\
\hline
\end{tabular}

Table 2 Additional parameters for power equation

\begin{tabular}{ccc}
\hline \hline Parameter & Description & Value \\
\hline$K=1 / \pi e_{0} A R$ & Aerodynamic parameter & 0.0347 \\
$A R=b^{2} / S$ & Aspect ratio & 9.6628 \\
$W=g m$ & Weight & $112.7 \mathrm{~N}$ \\
$\rho=\rho^{s}$ & Air density & $1.225 \mathrm{~kg} / \mathrm{m}^{3}$ \\
\hline \hline
\end{tabular}

Table 3 Camera model parameters

\begin{tabular}{ccc}
\hline \hline Parameter & Description & Value \\
\hline$f$ & Focal length & $0.0046 \mathrm{~m}$ \\
$H_{\text {dist }}$ & Horizontal distance of image plane & $0.00361 \mathrm{~m}$ \\
$V_{\text {dist }}$ & Vertical distance of image plane & $0.00272 \mathrm{~m}$ \\
\hline \hline
\end{tabular}

\section{Results}

We investigated the impact and tradeoffs between objectives from both the cyber and physical systems with the goal of minimizing energy use and time while maximizing information (minimizing entropy). Our goal is to show that simultaneous consideration of cyber and physical cost terms can yield more capable missions than what would be possible from designing these two parts of the CPS individually. We first examine and analyze Pareto fronts of the cost function in Eq. (13) to gain insight into the tradeoffs from competing objectives. We select candidate points along the Pareto front of several plots representing true multi-objective optimization, and we use the corresponding $v$ and $r_{\tau}$ to compute associated costs of the mission. We then select weights $\beta_{p 1}, \beta_{p 2}, \beta_{c 1}$, and $\beta_{c 2}$ optimize the CPS single objective cost function in Eq. (13) and compare results with solution points selected from the Pareto fronts.

\section{A. Pareto Fronts}

Pareto front examination and analysis gives insight into the tradeoffs between competing objectives. Pareto front plots of $J_{p}\left[\right.$ Eq. (5)] and $J_{c}$ [Eq. (10)] can be seen in Fig. 6, where the black (darker) data points represent the Pareto front.

These curves show how the objectives for the physical- and cyber systems, individually, tradeoff respective costs. The plots in Fig. 6 follow their respective governing dynamical equations to produce the curves shown. For Fig. $\underline{6 a}$, the plot is dominated by the power curve, indicating we could expend similar amounts of energy, accomplishing our mission in very different lengths of time. Clearly, to achieve our minimum time objective, the front side of the power curve is more optimal, as indicated by the Pareto front. Because our entropy cost is a function of both $v$ and $r_{\tau}$, we have multiple points corresponding to a single cyber rate $r_{\tau}$. As a result, the velocities resulting in a higher entropy cost are dominated by those producing lower entropy.

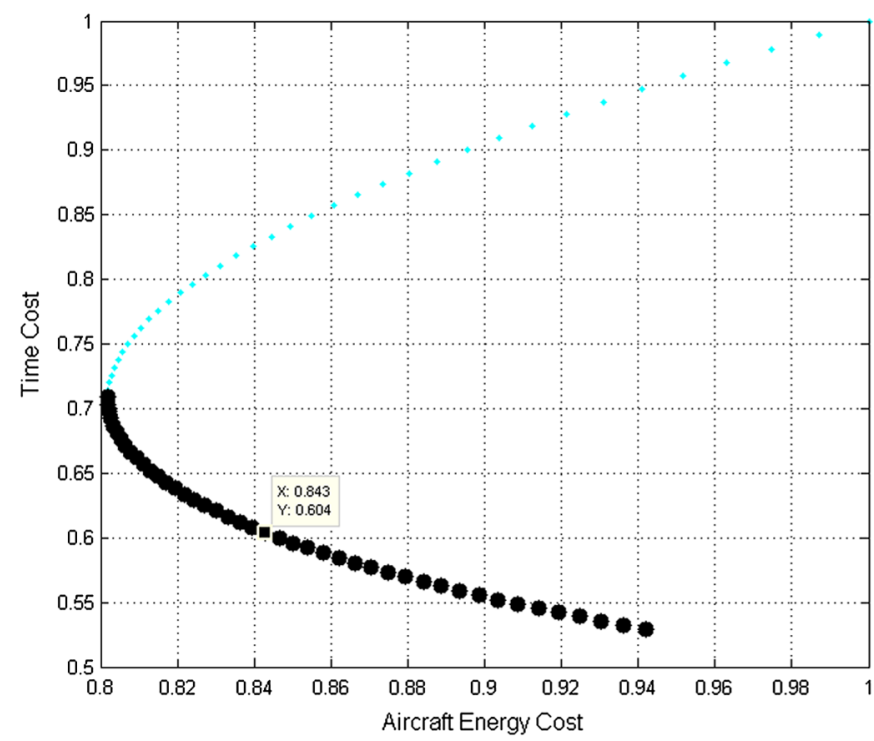

a) Pareto front for $J_{p}$

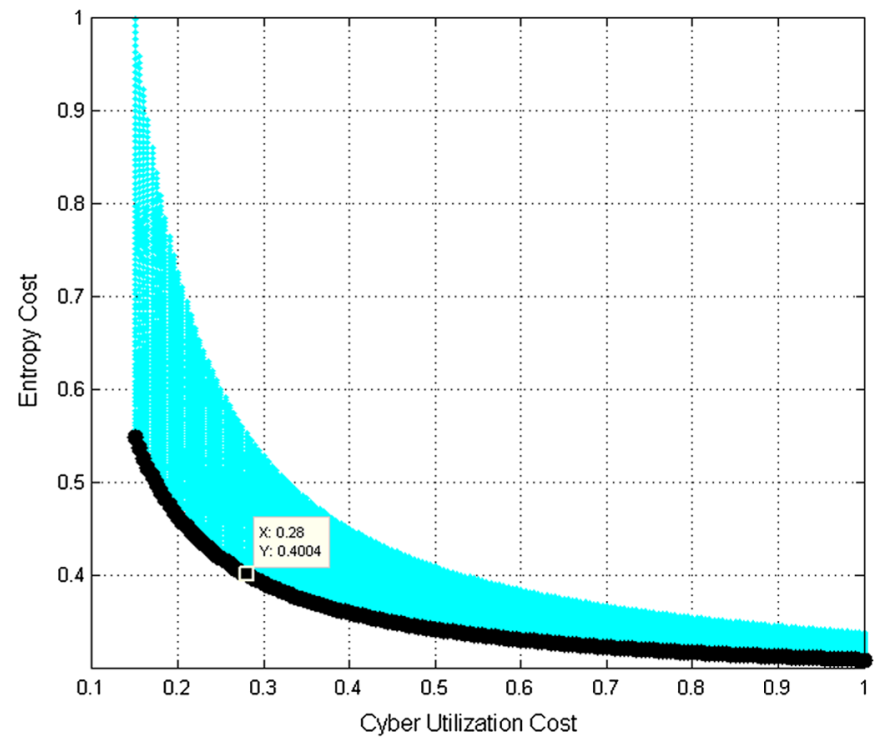

b) Pareto front for $J_{c}$

Fig. 6 Pareto fronts for $J_{p}$ and $J_{c}$. 
Table 4 Costs for $v=14.9 \mathrm{~m} / \mathrm{s}$ and $r_{\tau}=5.6 \mathrm{~Hz}$

\begin{tabular}{cc}
\hline \hline Parameters & Values \\
\hline$E$ & $16,626.2 \mathrm{~J}$ \\
$T$ & $134.2 \mathrm{~s}$ \\
$U_{\tau}$ & 0.28 \\
$H$ & 66.7 \\
\hline \hline
\end{tabular}

If we choose a solution along one of these Pareto fronts, we will optimize for either the physical or cyber portion of the system. Using these plots, we select the velocity corresponding with the data point highlighted in Fig. $6 \mathrm{a}: v=14.9 \mathrm{~m} / \mathrm{s}$. From the Pareto front for $J_{c}$, we choose the cyber rate corresponding with the data point highlighted in Fig. $\underline{6 \mathrm{~b}}$, or $r_{\tau}=5.6 \mathrm{~Hz}$. In Table $\underline{4}$, we show the costs associated with a mission using these parameters.

We can gain more insight into the tradeoffs of the entire cost function by also examining the tradeoffs between the CPS as a whole. We show these Pareto fronts in Fig. 7, where in each subfigure we examine the tradeoff between three of the four objectives. In Fig. 7a, we again observe the presence of the power curve governing the relationship between aircraft energy $E$ and the other objectives. The curve folds over onto itself, and we choose the point indicated in that plot that is in the crease of the function while also balancing cyber utilization $U_{\tau}$ and entropy $H$ costs.

In Fig. 7b, no new insight or information are gained, since the aircraft energy cost and total time cost $T$ are independent of the cyber utilization cost. Additionally, we note the similarity of this plot with the Pareto front for $J_{p}$ in Fig. 6 a . In the Pareto front plot in Fig. $7 \mathrm{c}$, there are no dominated points making the entire surface a Pareto front. We select the solution point indicated on this Pareto front that we determined from inspection of the plots, which provides appropriate balance between the competing objectives.

Figure 7d shows the tradeoffs between entropy, aircraft energy, and total time costs. In this Pareto front, we call attention to the normal tradeoff between total time and aircraft energy costs but, more interestingly, the tradeoff with entropy cost. This shows the coupling between cyber- and

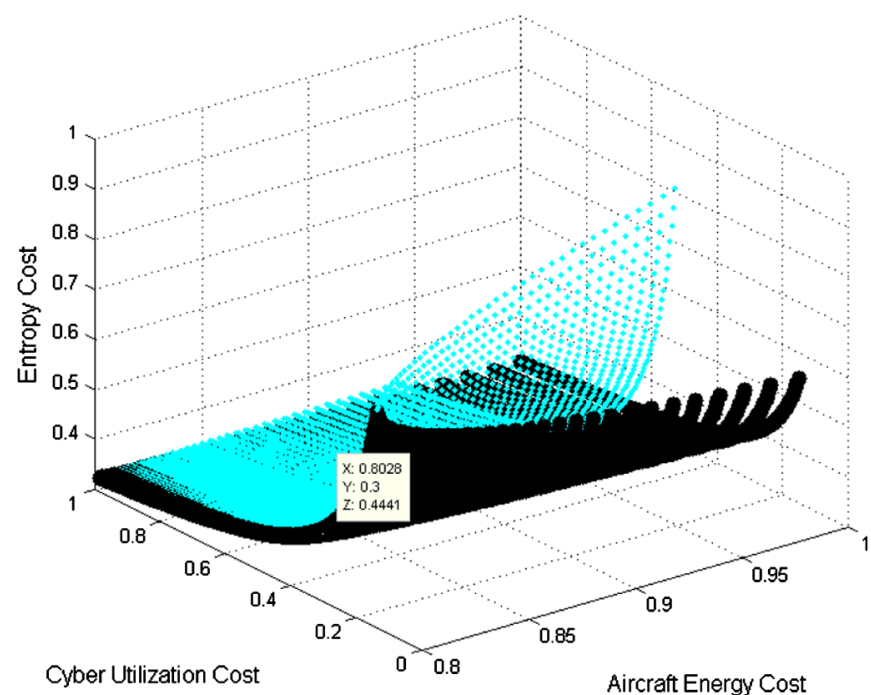

a) Pareto front for $E, U_{\tau}$, and $H$

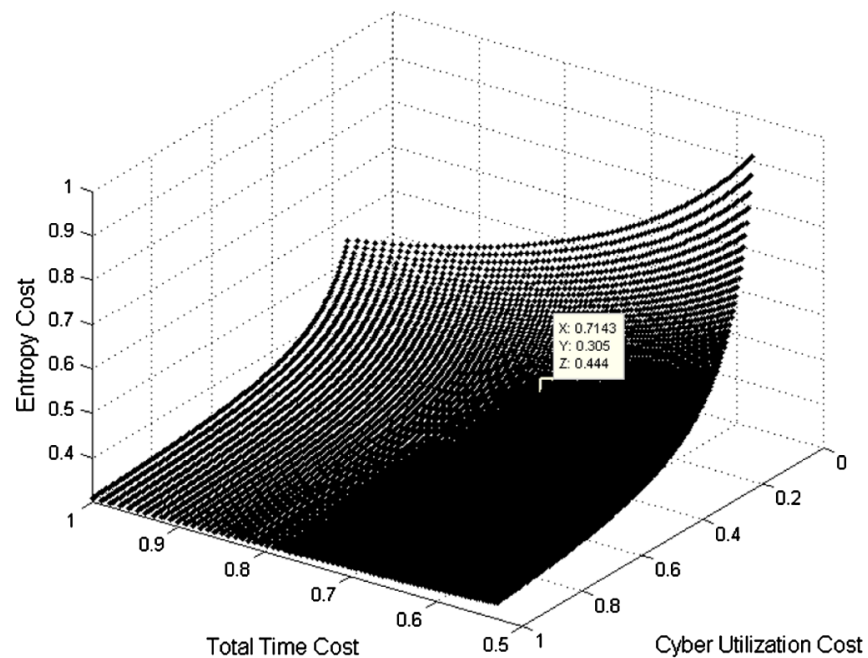

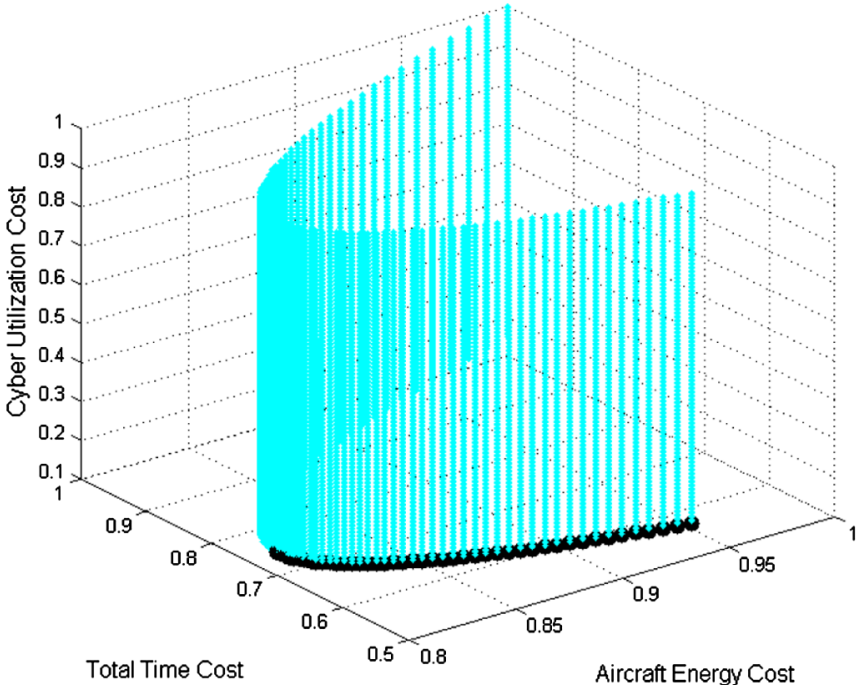

b) Pareto front for $E$, $T$, and $U_{\tau}$

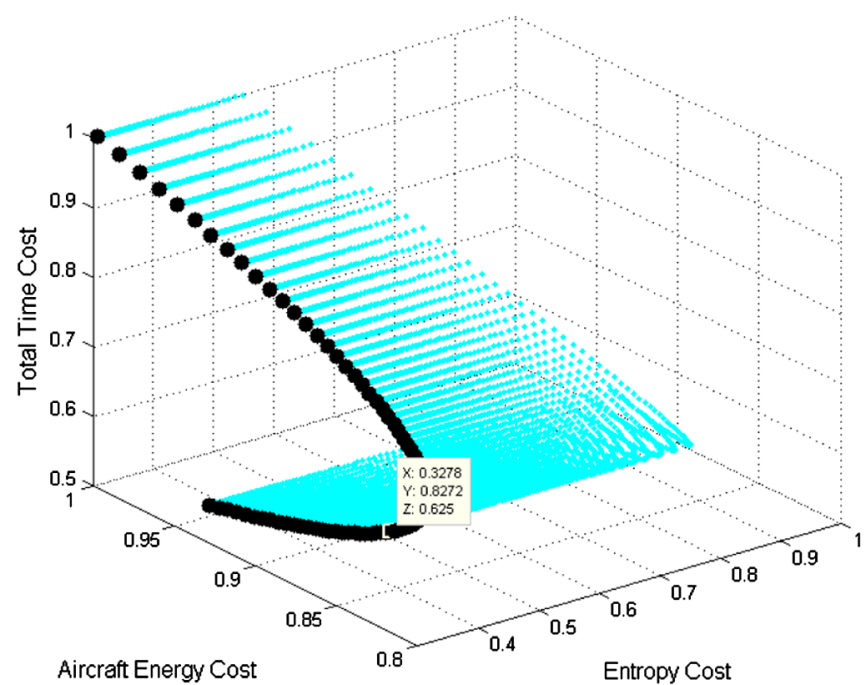

d) Pareto front for $H, T$, and $E$ 
Table 5 Parameters and costs for data points selected from Pareto fronts

\begin{tabular}{lcccc}
\hline \multicolumn{1}{c}{ Parameters } & $E, \mathrm{~J}$ & $T, \mathrm{~s}$ & $U_{\tau}$ & $H$ \\
\hline$v=14.4 \mathrm{~m} / \mathrm{s}, r_{\tau}=15.4 \mathrm{~Hz}$ & $16,314.5$ & 138.9 & 0.77 & 45.2 \\
$v=12.4 \mathrm{~m} / \mathrm{s}, r_{\tau}=6 \mathrm{~Hz}$ & $15,833.3$ & 161.3 & 0.30 & 58.4 \\
$v=12.6 \mathrm{~m} / \mathrm{s}, r_{\tau}=6.1 \mathrm{~Hz}$ & $15,816.9$ & 158.7 & 0.31 & 58.4 \\
\hline \hline
\end{tabular}

Table 6 Comparison of all solutions

\begin{tabular}{lccccccc}
\hline \hline \multicolumn{1}{c}{ Parameters } & Solution type & $E, \mathrm{~J}$ & $T, \mathrm{~s}$ & $U_{\tau}$ & $H$ & Total & \% worse than lowest cost solution \\
\hline$v=14.9 \mathrm{~m} / \mathrm{s}, r_{\tau}=5.6 \mathrm{~Hz}$ & Pareto from Table & $16,626.2$ & 134.2 & 0.28 & 66.7 & 0.5587 & 0.25 \\
$v=14.4 \mathrm{~m} / \mathrm{s}, r_{\tau}=15.4 \mathrm{~Hz}$ & Pareto from Table $\overline{5}$ & $16,314.5$ & 138.9 & 0.77 & 45.2 & 0.6416 & 15.13 \\
$v=12.4 \mathrm{~m} / \mathrm{s}, r_{\tau}=6 \mathrm{~Hz}$ & Pareto from Table $\frac{5}{5}$ & $15,833.3$ & 161.3 & 0.30 & 58.4 & 0.5682 & 1.96 \\
$v=12.6 \mathrm{~m} / \mathrm{s}, r_{\tau}=6.1 \mathrm{~Hz}$ & Pareto from Table & $15,816.9$ & 158.7 & 0.31 & 58.4 & 0.5663 & 1.61 \\
$v=15.2 \mathrm{~m} / \mathrm{s} r_{\tau}=18 \mathrm{~Hz}$ & $J$ with $\beta_{p 1}=\beta_{p 2}=0.5 \beta_{c 1}=\beta_{c 2}=0.0$ & $16,844.1$ & 131.6 & 0.90 & 44.3 & 0.6708 & 20.37 \\
$v=9.0 \mathrm{~m} / \mathrm{s} r_{\tau}=4.3 \mathrm{~Hz}$ & $J$ with $\beta_{p 1}=\beta_{p 2}=0.0 \beta_{c 1}=\beta_{c 2}=0.5$ & $19,722.8$ & 222.2 & 0.22 & 58.7 & 0.6654 & 19.40 \\
$v=14.2 \mathrm{~m} / \mathrm{s} r_{\tau}=5.6 \mathrm{~Hz}$ & $J$ with $\beta_{p 1}=\beta_{p 2}=\beta_{c 1}=\beta_{c 2}=0.25$ & $16,208.8$ & 140.8 & 0.28 & 64.9 & 0.5573 & Not applicable \\
\hline \hline
\end{tabular}

physical-cost terms and gives insight into how they compete in the total cost. We follow our previous reasoning in choosing a point that compromises total time and aircraft energy, but augmented by an attempt to minimize entropy as well.

We list the velocities, cyber rates, and corresponding costs for each of these three selected points in Table $\underline{5}$.

\section{B. Optimization over Total Cost Function $J\left(v, r_{\tau}\right)$}

In addition to examining Pareto fronts, using numerical methods, we can solve the single objective cost function $J\left(v, r_{\tau}\right)$ in Eq. (13), examine the resulting costs, and compare them with those found from the Pareto front analysis. This requires we select the weights for each cost term. Often, there are auxiliary reasons for favoring one cost term over another, such as length of time since the last mission or a cloudy day with less direct sunshine, which might result in a tighter energy budget for our solar-powered glider. Since we wish to investigate the comparison of holistic CPS optimization to independent physical- and cyber-system optimization, we allow corresponding weights to go to zero, as indicated in the fifth and sixth rows of Table 6 . In each case, however, in the absence of any compelling reasons to favor one term over another, we equalize all nonzero cost terms as shown. We compare the previous results from our Pareto analysis with our numerical solutions and show the individual costs, as well as the scaled and normalized total costs in Table 6 . The lowest total cost solution is the last entry in the table, wherein each individual cost term was given equal weight, and we compare its total cost with the other solutions as a percentage.

This paper has focused on a simulation-based analysis of CPS optimization for a UAS. An actual flight test demonstrating results was beyond the scope of this project. We have, however, been able to validate the basic CPS cost tradeoffs introduced in this paper using a single degree-offreedom satellite simulator (TableSat) in a laboratory environment [34].

\section{Conclusions}

As technology allows us to shrink physical platform size, resource use by a cyber-system (e.g., the computational and communication components) begins to rival actuation effort of the physical system required for propulsion and servo actuation. This paper investigates holistic optimization over both, demonstrating by example that codesign of the cyber-physical system results in a net savings of energy for given mission time and information gain by efficiently allocating cyber-physical system (CPS) resources.

Such a coupled codesign has been demonstrated in the form of optimization over cost functions, describing competing cyber and physical objectives. Unmanned aircraft system surveillance of a straight segment of pipeline was proposed as a baseline candidate mission, and simulation results were obtained. Pareto fronts of these results were analyzed, illustrating the presence of important tradeoffs between aircraft airspeed and task-execution rate for the candidate mission task. Optimal solutions were found to the combined CPS cost function, and they were compared to those results with independently optimized cyber and physical cost functions, demonstrating that large efficiency improvements can be realized by such an approach.

An important future enhancement to this work will be to demonstrate a dynamic real-time planner that can appropriately adapt cost function parameters that, in turn, modify the flight plan to maintain optimal conditions based on feedback from sensors, onboard data-processing elements, and mission operators. Examination of more complex missions will require additional CPS cost metrics; it is anticipated that this will promote innovations in measuring task, cyber, and overall mission success. Complex missions could involve overflight of rugged terrain, target tracking, and interaction with other cooperative, non-cooperative, or hostile entities. Results from real-world flight operations would also bolster this work, enabling evaluation of static or ultimately dynamic optimization to improve overall CPS metrics during representative missions such as area coverage or inspection.

\section{References}

[1] Cambone, S., Krieg, K., Pace, P., and Linton, W., “Unmanned Aircraft Systems Roadmap 2005-2030,” Office of the Secretary of Defense, U.S. Dept. of Defense, Aug. 2005.

[2] Andersson, J., “A Survey of Multiobjective Optimization in Engineering Design,” Dept. of Mechanical Engineering, Linköping Univ., Linköping, Sweden, 2000.

[3] Fontanelli, D., Greco, L., and Bicchi, A., "Anytime Control Algorithms for Embedded Real-time Systems," Hybrid Systems: Computation and Control, Springer, Berlin, 2008, pp. 158-171.

[4] Gupta, V., "On an Anytime Algorithm for Control," Proceedings of the 48th IEEE Conference on Decision and Control, IEEE, Piscataway, NJ, 2010, pp. 6218-6223. 
[5] Bhattacharya, R., and Balas, G., "Anytime Control Algorithm: Model Reduction Approach," Journal of Guidance, Control, and Dynamics, Vol. 27, No. 5, 2004, pp. 767-776. doi: $10.2514 / 1.9457$

[6] Sha, L., Abdelzaher, T., Årzén, K., Cervin, A., Baker, T., Burns, A., Buttazzo, G., Caccamo, M., Lehoczky, J., and Mok, A., "Real-Time Scheduling Theory: A Historical Perspective," Real-Time Systems, Vol. 28, No. 2, 2004, pp. 101-155. doi:10.1023/B:TIME.0000045315.61234.1e

[7] Arzén, K., Cervin, A., Eker, J., and Sha, L., "An Introduction to Control and Scheduling Co-Design," Proceedings of the 39th IEEE Conference on Decision and Control, Vol. 5, IEEE, Piscataway, NJ, 2002, pp. 4865-4870.

[8] Eker, J., Hagander, P., and Årzén, K., "A Feedback Scheduler for Real-Time Control Tasks," Control Engineering Practice, Vol. 8, No. 12, 2000, pp. 1369-1378. doi:10.1016/S0967-0661(00)00086-1

[9] Harrison, S., Price, M., and Philpott, M., "Task Scheduling for Satellite Based Imagery," Proceedings of the Eighteenth Workshop of the UK Planning and Scheduling Special Interest Group, Vol. 78, Univ. of Salford, U.K., 1999, pp. 64-78.

[10] Bataille, N., Lemaitre, M., and Verfaillie, G., "Efficiency and Fairness when Sharing the Use of a Satellite," Artificial Intelligence, Robotics and Automation in Space, Vol. 440, European Space Agency, Paris, 1999, p. 465.

[11] Bresina, J. L., Morris, R. A., and Edgington, W. R., "Optimizing Observation Scheduling Objectives," Proceedings of the 1997 NASA Workshop on Planning and Scheduling for Space, 1997.

[12] Chien, S., Sherwood, R., Tran, D., Cichy, B., Rabideau, G., Castano, R., Davies, A., Lee, R., Mandl, D., Frye, S., Trout, B., Hengemihle, J., D’ Agostino, J., Shulman, S., Ungar, S., Brakke, T., Boyer, D., Gaasbeck, J. V., Greeley, R., Doggett, T., Baker, V., Dohm, J., and Ip, F., "The EO-1 autonomous science agent," Proceedings of the Third International Joint Conference on Autonomous Agents and Multiagent Systems-Volume 1, IEEE Computer Society, Piscataway, NJ, 2004, pp. 420-427.

[13] Agrawal, M., Cofer, D., and Samad, T., "Real-Time Adaptive Resource Management for Advanced Avionics," IEEE Control Systems, Vol. 23, No. 1, 2003, pp. $76-86$. doi:10.1109/MCS.2003.1172831

[14] Abdelzaher, T. F., Atkins, E., and Shin, K., "QOS Negotiation in Real-Time Systems and its Application to Automated Flight Control," IEEE Transactions on Computers, Vol. 49, No. 11, 2000, pp. 1170-1183. doi: $10.1109 / 12.895935$

[15] Atkins, E., and Sanner, R., "QOS Tradeoffs for Guidance, Navigation, and Control,” 2002 IEEE Aerospace Conference Proceedings, Vol. 7, IEEE, Montana, 2002.

[16] Russ, M., and Stutz, P., "Airborne Sensor and Perception Management: A Conceptual Approach for Surveillance UAS," 2012 15th International Conference on Information Fusion (FUSION), IEEE, Singapore, July 2012, pp. 2444-2451.

[17] Narayan, P., Campbell, D., and Walker, R., "Computationally Adaptive Multi-Objective Trajectory Optimization for UAS with Variable Planning Deadlines," 2009 IEEE Aerospace Conference, IEEE, Piscataway, NJ, March 2009, pp. 1-8.

[18] Kirk, D., Optimal Control Theory: An Introduction, Dover, New York, 2004, p. 3.

[19] Bradley, J., and Atkins, E., "Toward Continuous State-Space Regulation of Coupled Cyber-Physical Systems," Proceedings of the IEEE, Vol. 100, No. 1, 2012, pp. 60-74. doi:10.1109/JPROC.2011.2161239

[20] McClamroch, N., Steady Aircraft Flight and Performance, Princeton Univ Press, Princeton, NJ, 2011, pp. 51, 97.

[21] Zhan, P., Casbeer, D., and Swindlehurst, A., "A Centralized Control Algorithm for Target Tracking with UAVs," 39th IEEE Asilomar Conference, Vol. 13, IEEE, Piscataway, NJ, 2005, p. 109

[22] Sinha, A., Kirubarajan, T., and Bar-Shalom, Y., "Autonomous Ground Target Tracking by Multiple Cooperative UAVs," 2005 IEEE Aerospace Conference, IEEE, Piscataway, NJ, March 2005, pp. 1-9.

[23] Poovendran, R., "Cyber-Physical Systems: Close Encounters Between Two Parallel Worlds," Proceedings of the IEEE, Vol. 98, No. 8, 2010, pp. 1363-1366. doi:10.1109/JPROC.2010.2050377

[24] Bradley, J. M., and Atkins, E. M., "Computational-Physical State Co-Regulation in Cyber-Physical Systems," ACM/IEEE Conference on Cyber-Physical Systems, ACM/IEEE, Chicago, IL, April 2011.

[25] Krishna, C., Real-Time Systems, Wiley Online Library, New York, 1999.

[26] Shannon, C. E., "A Mathematical Theory of Communication," The Bell System Technical Journal, Vol. 27, 1948, pp. 379-423, 623-656. doi:10.1145/584091.584093

[27] MacKay, D., Information Theory, Inference and Learning Algorithms, Cambridge Univ. Press, New York, 2003, pp. 67-84.

[28] Maes, F., Collignon, A., Vandermeulen, D., Marchal, G., and Suetens, P., "Multimodality Image Registration by Maximization of Mutual Information," IEEE Transactions on Medical Imaging, Vol. 16, No. 2, 1997, pp. 187-198. doi:10.1109/42.563664

[29] Gull, S., and Skilling, J., "Maximum Entropy Method in Image Processing," IEE Proceedings F: Communications, Radar, and Signal Processing, Vol. 131, No. 6, 1984, pp. 646-659. doi:10.1049/ip-f-1.1984.0099

[30] Lai, J., "A Hidden Markov Model and Relative Entropy Rate Approach to Vision-Based Dim Target Detection for UAV Sense-and-Avoid", Ph.D. Thesis, Queensland Univ. of Technology, Queensland, Australia, 2010.

[31] Ready, B., Taylor, C., and Beard, R., "A Kalman-Filter Based Method for Creation of Super-Resolved Mosaicks," Proceedings of the 2006 IEEE International Conference on Robotics and Automation: ICRA 2006, IEEE, Piscataway, NJ, May 2006, pp. 3417-3422.

[32] Hartley, R., and Zisserman, A., Multiple View Geometry in Computer Vision, Cambridge Univ. Press, New York, 2004, p. 154.

[33] Ma, Y., Soatto, S., Kosecka, J., and Sastry, S. S., An Invitation to 3-D Vision: From Images to Geometric Models, Springer-Verlag, New York, 2004, pp. 36-54.

[34] Bradley, J., Clark, M., Atkins, E., and Shin, K., "Mission-Aware Cyber-Physical Optimization on a Tabletop Satellite," Infotech@Aerospace, AIAA Paper 2013-4807, 2013. 